\title{
ON THE LEAST SQUARES SIGNAL APPROXIMATION MODEL FOR OVERDECIMATED RATIONAL NONUNIFORM FILTER BANKS AND APPLICATIONS
}

\author{
Andre Tkacenko and P. P. Vaidyanathan \\ Dept. of Electrical Engineering \\ California Institute of Technology, Pasadena, CA 91125, USA \\ E-mail: andre@systems.caltech.edu,ppvnath@systems.caltech.edu
}

\begin{abstract}
With the advent of wavelets for lossy data compression came the notion of representing signals in a certain vector space by their projections in well chosen subspaces of the original space. In this paper, we consider the subspace of signals generated by an overdecimated rational nonuniform filter bank and find the optimal conditions under which the mean-squared error between a given deterministic signal and its representation in this subspace is minimized for a fixed set of synthesis filters. Under these optimal conditions, it is shown that choosing the synthesis filters to further minimize this error is simply an energy compaction problem. With this, we introduce the notion of deterministic energy compaction filters for classes of signals. Simulation results are presented showing the merit of our proposed method for optimizing the synthesis filters. ${ }^{1}$
\end{abstract}

\section{INTRODUCTION}

Along with the introduction of wavelets in the field of signal processing came the notion of representing signals in a given vector space, usually $\ell_{2}$, by their projections in certain special types of subspaces of the original space. The impetus for such a representation arises in lossy data compression and multiresolution theory $[3,4]$, since the above projections often require less information to be stored than the original signal itself at the cost of a small amount of loss of fidelity.

'In this paper, we consider the subspace of signals generated by an overdecimated rational nonuniform synthesis bank as shown in Figure 1. By overdecimated, we mean that,

$$
\sum_{k=0}^{P-1} \frac{m_{k}}{n_{k}}<1
$$

and so the inputs $\left\{c_{k}(n)\right\}$ operate at a lower overall rate than the output $y(n)$. The subspace $\mathcal{V}$ that we will focus on is defined by,

$$
\mathcal{V}^{\prime} \triangleq\left\{y(n): y(n)=\sum_{k=0}^{P-1} \sum_{m=-\infty}^{\infty} c_{k}(m) f_{k}\left(m_{k} n-n_{k} m\right)\right\}
$$

For a fixed set of synthesis filters $\left\{F_{k}(z)\right\}$, we will find the optimal choice of the driving signals $\left\{c_{k}(n)\right\}$ which minimize the meansquared error between $y(n)$ and any given signal $x(n) \in \ell_{2}$. This

\footnotetext{
${ }^{1}$ Work supported in part by the ONR grant N00014-99-1-1002, USA.
}

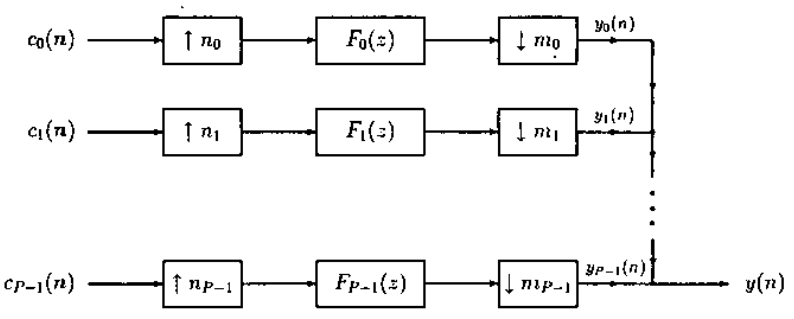

Fig. 1. Rational nonuniform synthesis filter bank.

is a generalization of the results given in [7] for integer nonuniform filter banks. Though rational nonuniform filter banks can be shown to be transformable to integer nonuniform filter banks, our approach avoids this complicated transformation and solves the least squares problem in a more direct way.

In addition to solving this least squares problem, we also consider optimizing the synthesis filters to further minimize the meansquared error. This is shown to be analogous to choosing the optimum filter for compacting the energy of a process whose power spectrum is related to the blocked version of the given signal $x(n)$. Though for a single deterministic signal, we can trivially force the mean-squared error to be zero through proper choice of the synthesis filters, the filters here are chosen to minimize the mean-squared error for a class of deterministic signals with certain practical constraints. With this, we introduce the notion of deterministic energy compaction filters for classes of signals. Experimental results provided here show the merit of our proposed method.

\subsection{Notations}

All notations are as in [6] unless specified otherwise. In particular, $M$-fold decimation and expansion will be represented by the symbols $\downarrow M$ and $\uparrow M$, respectively. Also, the $M$-fold blocked version of a scalar signal $x(n)$ is an $M \times 1$ vector signal $\mathbf{x}(n)$ given by,

$$
\mathbf{x}(n) \triangleq\left[\begin{array}{c}
x(M n) \\
x(M n+1) \\
\vdots \\
x(M n+(M-1))
\end{array}\right]
$$

Finally, the $M$-fold blocked representation of a scalar transfer function $H(z)$ is an $M \times M$ multiple-input multiple-output (MIMO) pseudocirculant system $\mathbf{H}(z)$ whose 0-th column consists of the Type 1 polyphase components of $H(z)$. 


\section{LEAST SQUARES SIGNAL APPROXIMATION MODEL}

Consider the rational nonuniform synthesis filter bank shown in Figure 1. We will make the following assumptions here.

- $\operatorname{gcd}\left(m_{k}, n_{k}\right)=1 \forall k$ (Coprimeness of $m_{k}$ and $n_{k}$ )

- $\sum_{k=0}^{P-1} \frac{m_{k}}{n_{k}}<1$

(Overdecimated system)

There is no loss of generality in making the first assumption, as common factors between $m_{k}$ and $n_{k}$ can be absorbed into the filter $F_{k}(z)$. The second assumption ensures that the subspace $\mathcal{V}$ in (1) is a proper subspace of $\ell_{2}$. Let us define the following integers.

- $N \triangleq \operatorname{lcm}\left(n_{0}, n_{1}, \ldots, n_{P-1}\right)$

- $p_{k} \triangleq \frac{N}{n_{k}} \forall k$

- $K \triangleq \sum_{k=0}^{P-1} m_{k} p_{k}$

Note that as the system is overdecimated, we have $K<N$.

The goal here is to choose the driving signals $\left\{c_{k}(n)\right\}$ to minimize the mean-squared error objective,

$$
\xi \triangleq \sum_{n}|y(n)-x(n)|^{2}
$$

where $x(n)$ is any signal in $\ell_{2}$. If $\mathbf{x}(n)$ and $\mathbf{y}(n)$ denote, respectively, the $N$-fold blocked versions of $x(n)$ and $y(n)$, we have,

$$
\xi=\sum_{n}\|\mathbf{y}(n)-\mathbf{x}(n)\|^{2}
$$

Using Parseval's relation, this in turn can be expressed as follows.

$$
\xi=\frac{1}{2 \pi} \int_{0}^{2 \pi}\left\|\mathbf{Y}\left(e^{j \omega}\right)-\mathbf{X}\left(e^{j \omega}\right)\right\|^{2} d \omega
$$

where $\mathbf{X}(z)$ and $\mathbf{Y}(z)$ denote, respectively, the $z$-transforms of $\mathbf{x}(n)$ and $\mathbf{y}(n)$.

To simplify $\mathrm{Y}(z)$, consider the $k$-th branch of the system of Figure 1 reproduced in Figure 2(a). If we implement $F_{k}(z)$ in an $m_{k} N$-fold block form, we obtain the system shown in Figure 2(b), where $\mathbf{A}_{k}(z)$ is an $m_{k} N \times m_{k} N$ pseudocirculant matrix [6] with,

$$
\left[\mathbf{A}_{k}(z)\right]_{r, s}=\left[z^{r-s} F_{k}(z)\right]_{\downarrow m_{k} N}
$$

for $0 \leq r, s \leq m_{k} N-1$. By applying the polyphase identity [6], the expander on the left $\left(\uparrow n_{k}\right)$ as well as the decimator on the right ( $\downarrow m_{k}$ ) can be moved across the network resulting in the system of Figure 2(c). The $N \times m_{k} p_{k}$ transfer matrix $\mathbf{F}_{k}(z)$ is obtained by preserving only the $N$ rows of $\mathbf{A}_{k}(z)$ which are multiples of $m_{k}$ and the $m_{k} p_{k}$ columns which are multiples of $n_{k}$. In other words,

$\left[\mathbf{F}_{k}(z)\right]_{c, d}=\left[z^{c m_{k}-d n_{k}} F_{k}(z)\right]_{\downarrow m_{k} N}=\left[z^{c}\left[z^{-d n_{k}} F_{k}(z)\right]_{\downarrow m_{k}}\right]$

for $0 \leq c \leq N-1$ and $0 \leq d \leq m_{k} p_{k}-1$. Note that from Figure 2(c), $c_{k}(n)$ is simply the $m_{k} p_{k}$-fold blocked version of $c_{k}(n)$ and $\mathbf{y}_{k}(n)$ is the $N$-fold blocked version of $y_{k}(n)$. Clearly, we have,

$$
\mathbf{Y}_{k}(z)=\mathbf{F}_{k}(z) \mathbf{C}_{k}(z)
$$

But note that we have,

$$
y(n)=\sum_{k=0}^{P-1} y_{k}(n) \Longleftrightarrow \mathbf{y}(n)=\sum_{k=0}^{P-1} \mathbf{y}_{k}(n)
$$

Thus, using (4), we get,

$$
\mathbf{Y}(z)=\sum_{k=0}^{P-1} \mathbf{Y}_{k}(z)=\sum_{k=0}^{P-1} \mathbf{F}_{k}(z) \mathbf{C}_{k}(z)
$$

This can be expressed as,

$$
\mathbf{Y}(z)=\underbrace{\left[\begin{array}{llll}
\mathbf{F}_{0}(z) & \mathbf{F}_{1}(z) & \cdots & \mathbf{F}_{P-1}(z)
\end{array}\right]}_{\mathbf{F}(z)} \underbrace{\left[\begin{array}{c}
\mathbf{C}_{0}(z) \\
\mathbf{C}_{1}(z) \\
\vdots \\
\mathbf{C}_{P-1}(z)
\end{array}\right]}_{\mathbf{C}(z)}
$$

where $\mathbf{F}(z)$ is an $N \times K$ matrix and $\mathbf{C}(z)$ is a $K \times 1$ vector. Note that even though the fixed matrix $\mathbf{F}(z)$ has a restricted structure as can be seen from (3), the vector $\mathbf{C}(z)$ is completely arbitrary.

Substituting (5) into (2), we have,

$$
\xi=\frac{1}{2 \pi} \int_{0}^{2 \pi}\|\underbrace{\mathbf{F}\left(e^{j \omega}\right) \mathbf{C}\left(e^{j \omega}\right)-\mathbf{X}\left(e^{j \omega}\right)}_{\boldsymbol{\epsilon}(\omega)}\|^{2} d \omega
$$

and so we can minimize $\xi$ by minimizing $\|\epsilon(\omega)\|^{2}$ pointwise in $\omega$. The solution to this well known least squares problem is [2],

$$
\mathbf{C}\left(e^{j \omega}\right)=\left[\mathbf{F}^{\dagger}\left(e^{j \omega}\right) \mathbf{F}\left(e^{j \omega}\right)\right]^{+} \mathbf{F}^{\dagger}\left(e^{j \omega}\right) \mathbf{X}\left(e^{j \omega}\right)
$$

where $\mathbf{A}^{+}$denotes the Moore-Penrose pseudoinverse of the matrix A [2]. We will assume here that $\mathbf{F}\left(e^{j \omega}\right)$ has a full rank of $K$ and so the pseudoinverse from above will in fact be a true inverse. In the $z$-domain, the optimum driving signal $\mathbf{C}(z)$ is given by,

$$
\mathbf{C}(z)=\underbrace{[\tilde{\mathbf{F}}(z) \mathbf{F}(z)]^{-1} \tilde{\mathbf{F}}(z)}_{\mathbf{H}(z)} \mathbf{X}(z)
$$

where $\widetilde{\mathbf{A}}(z) \triangleq \mathbf{A}^{\dagger}\left(1 / z^{*}\right)$ for any $\mathbf{A}(z)$ [6]. Hence, the optimal $C(z)$ from (6) can be obtained via the system shown in Figure 3.

\section{OPTIMIZING THE SYNTHESIS FILTERS SUBJECT TO A PARAUNITARY CONSTRAINT}

The optimal driving signal vector $\mathbf{C}(z)$ in (6) can be viewed as an information compacted version of the blocked signal vector $\mathbf{X}(z)$. In this setting, a signal to be approximated, say $x(n)$, will be processed through the network of Figure 3 to produce $\mathbf{C}(z)$ (or equivalently $\left.\left\{c_{k}(n)\right\}\right)$. The $\left\{c_{k}(n)\right\}$ are then stored and used to obtain $y(n)$, the best approximation to $x(n)$ for the given model.

Typically $x(n)$ will be a finite length signal (i.e. a speech signal or an image) and we want the signals $\left\{c_{k}(n)\right\}$ to be finite in length themselves. In addition, we also want the synthesis filters $\left\{F_{k}(z)\right\}$ to be finite impulse response (FIR) filters. However, in general, if these filters are FIR, then the transfer function $\mathbf{H}(z)$ used to obtain $\mathbf{C}(z)$ in (6) will have an infinite impulse response (IIR). This is due to the factor $[\widetilde{\mathbf{F}}(z) \mathbf{F}(z)]^{-1}$ present in $\mathbf{H}(z)$. 


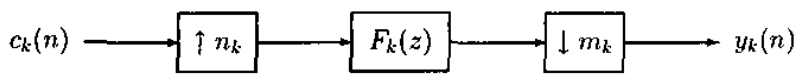

(a)

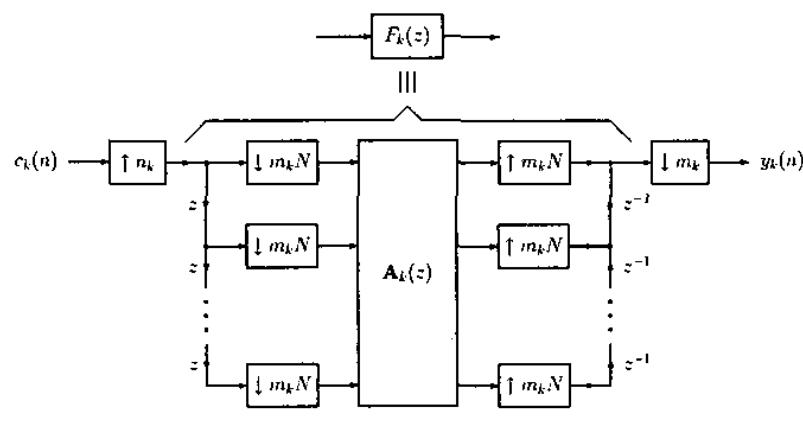

(b)

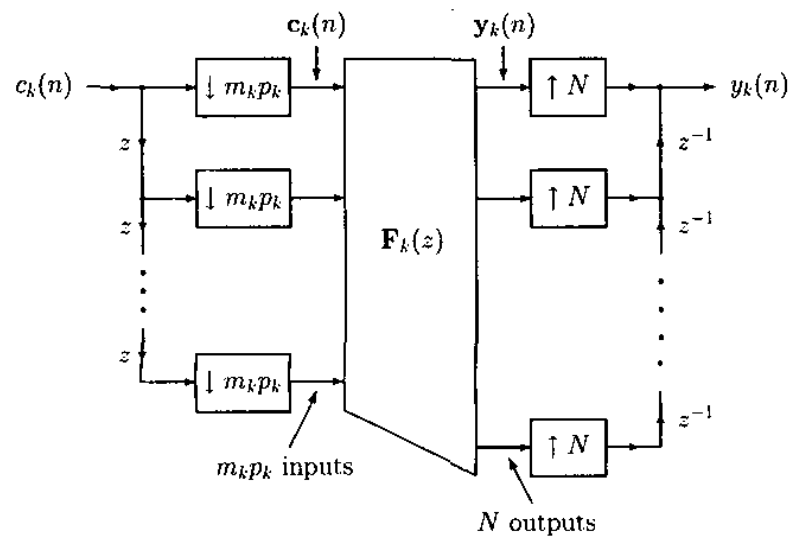

(c)

Fig. 2. (a) The $k$-th branch of the signal model, (b) With $F_{k}(z)$ implemented in an $m_{k} N$-fold block form, (c) Resulting structure after applying the polyphase identity.

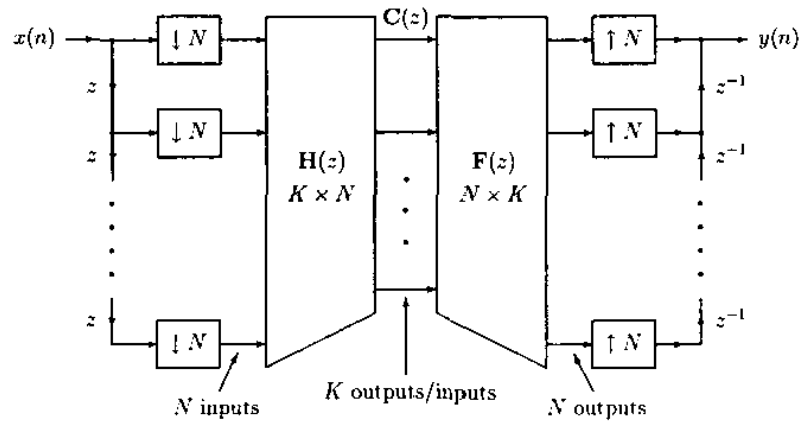

Fig. 3. System for obtaining the optimal driving $\operatorname{signal} \mathbf{C}(z)$.

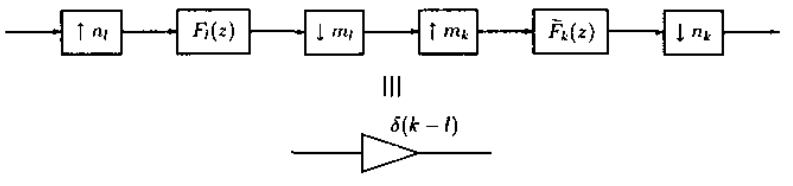

Fig. 4. Condition for orthonormality of the synthesis filters.

One way to $\operatorname{keep} \mathbf{H}(z)$ FIR and consequently to keep the driving signals $\left\{c_{k}(n)\right\}$ finite in length is to impose the paraunitary condition on $\mathbf{F}(z)$ [6]. Namely, we will constrain $\mathbf{F}(z)$ to satisfy,

$$
\widetilde{\mathbf{F}}(z) \mathbf{F}(z) \Rightarrow \mathbf{I}
$$

Given the structure inherent in $\mathbf{F}(z)$ (see (5) and (3)), it is not obvious that we can even satisfy (7). However, it turns out that (7) is satisfied iff the synthesis filters $\left\{F_{k}(z)\right\}$ form an orthonormal basis, as shown in Figure 4. This is stated formally in the following theorem, the proof of which is omitted for sake of brevity.

Theorem 1 The transfer function $\mathrm{F}(z)$ given by (5) and (3) satisfies the paraunitary condition of (7) iff the synthesis filters $\left\{F_{k}(z)\right\}$ satisfy the orthonormality relation shown in Figure 4.

From [1], the orthonormality condition given in Figure 4 can be expressed algebraically as,

$$
\sum_{n} f_{k}^{*}\left(m_{k} n-n_{k} m\right) f_{l}\left(m_{l} n-n_{l} i\right)=\delta(k-l) \delta(m-i)
$$

Hence, the paraunitary condition for $\mathbf{F}(z)$ in (7) is equivalent to the orthonormality condition in (8). It should be noted that the condition given in (8) can often be satisfied in practice and so indeed we can usually ensure the paraunitarity of $\mathbf{F}(z)$ as desired.

\section{RELATION TO ENERGY COMPACTION}

Using the optimal $\mathbf{C}(z)$ of (6), the error $\xi$ can be expressed as,

$\xi=\sum_{n}|x(n)|^{2}-\underbrace{\int_{0}^{2 \pi} \operatorname{Tr}\left[\mathbf{G}\left(e^{j \omega}\right) \mathbf{X}\left(e^{j \omega}\right) \mathbf{X}^{\dagger}\left(e^{j \omega}\right) \mathbf{G}^{\dagger}\left(e^{j \omega}\right)\right] \frac{d \omega}{2 \pi}}_{\sigma^{2}}$

where $\mathbf{G}(z) \triangleq[\widetilde{\mathbf{F}}(z) \mathbf{F}(z)]^{-\frac{1}{2}} \tilde{\mathbf{F}}(z)$. Hence, minimizing $\xi$ is equivalent to maximizing $\sigma^{2}$. But maximizing $\sigma^{2}$ is equivalent to compacting the energy of a filtered wide sense stationary (WSS) process $\mathbf{V}(z) \triangleq \mathbf{G}(z) \mathbf{W}(z)$ where $\mathbf{W}(z)$ is WSS with a power spectral density (psd) of $\mathbf{S}_{\mathbf{w w}}(z)=\mathbf{X}(z) \widetilde{\mathbf{X}}(z)$. In the single channel integer case, it was shown [5] that $\xi$ could be made zero by trivially adjusting the subspace to accomodate the given deterministic signal $x(n)$. To avoid such trivialities, Unser considered the energy compaction problem for an ensemble of signals characterized by a WSS process $x(n)$ with a psd of $S_{x x}(z)$ subject to the paraunitary constraint of (7) for the single channel integer case.

In this paper, we consider the energy compaction problem for a collection or class of deterministic signals. The class may represent, for example, a set of vowel phonemes uttered by a variety of speakers, or a set of images with a common theme. Consider a set of $L$ signals $\left\{x_{l}(n)\right\}$ for $0 \leq l \leq L-1$. Using the optimal driving signal model from Figure 3, we propose to choose $\mathbf{F}(z)$ subject to the paraunitary constraint in (7) to minimize the objective,

$$
J \triangleq \sum_{l=0}^{L-1} \alpha_{l} \xi_{l} \text { where } \xi_{l} \triangleq \sum_{n}\left|y_{l}(n)-x_{l}(n)\right|^{2}
$$



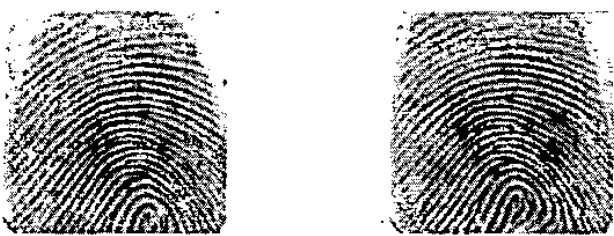

$x_{0}(n)$

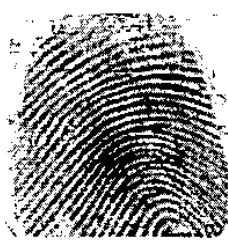

$x_{2}(n)$ $x_{1}(n)$

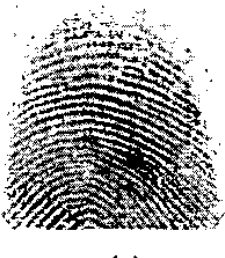

$x_{3}(n)$

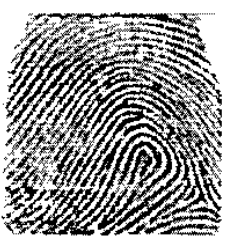

(a)

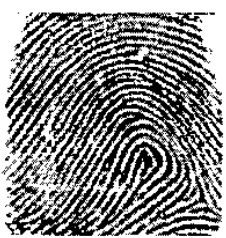

(c)

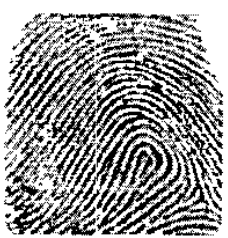

(b)

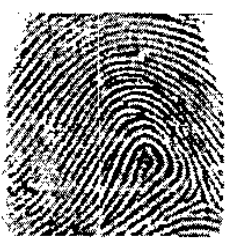

(d)

Fig. 5. Collection of images used for simulations.

and $y_{l}(n)$ is the output of $x_{l}(n)$ to the system of Figure 3. Here, $\alpha_{l}$ is a weighing factor that satisfies,

$$
\alpha_{l} \geq 0, \sum_{l=0}^{L-1} \alpha_{l}=1
$$

With this, it can be shown that choosing $\mathbf{F}(z)$ to maximize $J$ is equivalent to the above energy compaction problem with,

$$
\mathbf{S}_{\mathbf{w w}}(z)=\sum_{l=0}^{L-1} \alpha_{l} \mathbf{X}_{l}(z) \tilde{\mathbf{X}}_{l}(z)
$$

\section{SIMULATION RESULTS}

To test our proposed design method, we used it for a set of $L=$ 4 images shown in Figure 5. Each image is from a fingerprint database and the goal is to design an optimal synthesis bank for the class of such images. The images were converted to onedimensional signals via a raster scan. Equal weighing was used for each signal (i.e. $\alpha_{l}=\frac{1}{L} \forall l$ ). Minimizing $J$ in (9) subject to (7) is equivalent to maximizing a quadratic form subject to quadratic constraints. As such, nonlinear optimization techniques were used.

In order to see the merit of our approach, we applied a model signal, shown in Figure 6(a), to a variety of our optimally designed synthesis banks. We considered the following filter banks.

- (Integer nonuniform) $P=2, m_{k}=1 \forall k, n_{0}=2, n_{1}=4$

- (Uniform) $P=3, m_{k}=1, n_{k}=4 \forall k$

- (Integer single channel) $P=1, m_{0}=1, n_{0}=2$

For simplicity, we chose the length of all filters to be 4 . The results of applying the model signal to the above optimally designed filter banks are shown in Figure 6(b), (c), and (d), respectively.

From Figure 6, we can see that all methods yielded outputs similar in appearance to the given model signal, although the single channel case appears to have Moiré patterns when viewed at full size [8]. To quantitatively compare the methods, we calculated the peak signal-to-noise ratio (PSNR) of the output signals, which can be found in the caption of Figure 6 . Here, the uniform case performed the best, while the single channel case was the worst. Though the uniform case performed better than the nonuniform one, this was at the expense of having another channel. This brings to light the tradeoff between signal fidelity and computational load.

Fig. 6. (a) Original model signal, (b) Nonuniform case (PSNR $=40.18 \mathrm{~dB}$ ), (c) Uniform case (PSNR = 47.90 dB), (d) Single channel case (PSNR $=34.05 \mathrm{~dB}$ ).

\section{CONCLUDING REMARKS}

In this paper, we considered the least squares approximation model for rational nonuniform synthesis banks and showed the equivalence of the paraunitarity of the matrix $F(z)$ and the orthonormality of the synthesis filters. We also introduced the notion of deterministic compaction filters for a class or collection of signals and showed the merit of our method with examples. Future research includes studying the effects of quantizing the optimal driving signals $\left\{c_{k}(n)\right\}$ for the purpose of further compression.

\section{REFERENCES}

[1] T. Chen and P. P. Vaidyanathan, "Vector space framework for unification of one- and multidimensional filter bank theory," IEEE Trans. on Signal Processing, 42(8):2006-2021, Aug. 1994.

[2] R. A. Horn and C. R. Johnson, Matrix Analysis, Cambridge Univ. Press, Cambridge, U.K., 1985.

[3] S. G. Mallat, A Wavelet Tour of Signal Processing, Academic Press, London, U.K., 1999.

[4] S. G. Mallat, "A theory of multiresolution signal decomposition: The wavelet representation," IEEE Trans. on Patt. Anal. Mach. Intell., 11:674-693, 1989.

[5] M. Unser, "On the optimality of ideal filters for pyramid and wavelet signal approximation," IEEE Trans. on Signal Pro. cessing, 41(12):3591-3596, Dec. 1993.

[6] P. P. Vaidyanathan, Multirate Systems and Filter Banks, Prentice-Hall, Inc., Englewood Cliffs, NJ, 1993.

[7] B. Vrcelj and P. P. Vaidyanathan, "Least squares signal approximation using multirate systems: multichannel nonuniform case," in Proc. Asilomar Conf. Signals, Systems, and Computers, Monterey, CA, Nov. 2001, pp. 553-557.

[8] http://www.systems.caltech.edu/dsp/ students/andre/papers/models.pdf 